\title{
Aspectos clínicos e tratamento da amelogênese imperfeita: relato de caso
}

\author{
- Bruna Mandrá da Cunha Universidade de Uberaba (UNIUBE), Uberaba, MG, Brasil • Paula Guitarrara NirschI \\ Morais Nery Universidade de Uberaba (UNIUBE), Uberaba, MG, Brasil • Cristina Luzia Lopes Borges Silva \\ Universidade de Uberaba (UNIUBE), Uberaba, MG, Brasil • João Paulo Silva Servato Universidade de Uberaba \\ (UNIUBE), Uberaba, MG, Brasil • Maria Angélica Hueb de Menezes Oliveira Universidade de Uberaba (UNIUBE), \\ Uberaba, MG, Brasil • Denise Tornavoi de Castro Universidade de Uberaba (UNIUBE), Uberaba, MG, Brasil
}

RESUMO | Objetivo: Este trabalho versa sobre as características clínicas de um caso de amelogênese imperfeita (AI) do tipo IIA (hipomaturado com pigmentação difusa), apresentando formas de tratamento e como essa condição afeta a qualidade de vida do paciente. Materiais e métodos: Um paciente com 7 anos de idade, do sexo masculino, apresentando dentição decídua e permanente, procurou atendimento odontológico em razão das seguintes alterações dentárias: coloração dental amarelo-acastanhado, superfície rugosa, presença de cálculos dentais nos incisivos inferiores, mordida topo a topo e sensibilidade dentinária exacerbada. No exame radiográfico foi possível observar que o volume dos tecidos dentários estava normal, mas existia falta de contraste entre esmalte e dentina em todos os dentes, características compatíveis com o diagnóstico clínico de AI-IIA. Como tratamento, foram realizadas restaurações com cimento de ionômero de vidro, aplicação de flúor verniz e bochechos com fluoreto de sódio 0,05\% diariamente. Resultados: O tratamento executado foi considerado um sucesso, uma vez que as queixas do paciente foram solucionadas por meio de um tratamento odontológico adequado, restabelecendo a função e a estética da criança de acordo com sua necessidade. Conclusões: O diagnóstico precoce da AI é de suma importância para um tratamento mais conservador e com enfoque na prevenção dos efeitos dessa patologia. Do ponto de vista odontológico, o tratamento da AI tem como principal objetivo devolver a saúde bucal do paciente e, consequentemente, reintegrá-lo ao convívio social com maior qualidade de vida.

DESCRITORES | Amelogênese Imperfeita; Esmalte Dentário; Cimento de Ionômero de Vidro; Radiografia Panorâmica.

ABSTRACT | Clinical aspects and treatment of amelogenesis imperfect: A case report - Objectives: This study discusses the clinical characteristics of a case of type IIA amelogenesis imperfecta (hypomatured with diffuse pigmentation), presenting the treatment methods and how this condition affects the patient's quality of life. Materials and methods: A 7-year-old male patient with deciduous and permanent dentition sought dental care due to the following dental changes: brownishyellow dental coloration, rough surface, presence of dental calculus on the lower incisors, end-to-end bite, and dentin hypersensitivity. Radiographic examination showed that the volume of dental tissues was normal, but there was a lack of contrast between enamel and dentin in all teeth - features compatible with the clinical diagnosis of AI-IIA. Treatment consisted of restorations performed with glass ionomer cement, application of fluoride varnish and daily mouthwash with 0.05\% sodium fluoride. Results: The treatment performed was considered a success, since the patient's complaints were addressed through an appropriate dental treatment, restoring the child's function and aesthetics according to his needs. Conclusions: Early diagnosis of AI is of paramount importance for more conservative treatment focusing on preventing its effects. From the dental care point of view, AI treatment seeks to restore the patient's oral health and consequently reintegrate them into social life with a higher quality of life.

DESCRIPTORS | Amelogenesis Imperfecta; Dental Enamel; Glass Ionomer Cements; Radiography Panoramic.

AUTOR CORRESPONDENTE | • Denise Tornavoi de Castro Universidade de Uberaba • Av. Nenê Sabino, 1801 Uberaba, MG, Brasil • 38055-500 E-mail: denise.castro@uniube.br

• Recebido 26112021 • Aceito 19012022.

- DOI: http://dx.doi.org/10.11606/issn.2357-8041.clrd.2022.192922. 


\section{INTRODUÇÃO}

A amelogênese imperfeita (AI) é uma anomalia genética que provoca alterações na estrutura e nas características clínicas do esmalte dentário, podendo estar associada a diversas mudanças sistêmicas morfológicas e/ou bioquímicas. ${ }^{1}$ Existem 14 subtipos de AI, classificados de acordo com seus fenótipos (aparência clínica) e genótipos (herança hereditária), podendo se apresentar como distúrbios autossômico dominante, autossômico recessivo ou ligado ao cromossomo X. ${ }^{2}$

A Classificação Internacional de Doenças (CID) é vaga em relação à categorização dessa anomalia, não apresentando qualquer subdivisão. Apesar disso, diversos autores classificam o esmalte afetado pela AI em três fenótipo/formas clínicas distintas: hipoplásica, hipocalcificada e hipomaturada. Na AI do tipo hipoplásica, o esmalte apresenta a matriz orgânica defeituosa, o que o torna fino, com fissuras e sulcos, provocando a transparência e a exposição da dentina subjacente. $O$ tamanho dos dentes pode diminuir, o que provoca falta de contatos interproximais. No exame radiográfico é possível observar o contraste entre os tecidos dentais mineralizados, evidenciando a mineralização normal do esmalte afetado. ${ }^{1,3}$

No tipo hipocalcificada, a matriz orgânica tem espessura normal, mas a calcificação é deficiente e o esmalte se torna frágil/amolecido, escurecido e manchado. Ao erupcionar, os dentes apresentam forma e tamanho normais, porém, ao iniciar sua função, podem sofrer corrosões, modificando sua aparência inicial. Devido a calcificação deficiente, a radiopacidade do esmalte é menor, sendo difícil distingui-lo da dentina. ${ }^{4}$

$\mathrm{Na}$ forma hipomaturada, ocorrem defeitos na formação dos cristais de hidroxiapatita durante a fase de maturação, gerando um esmalte fino e rígido, que não se desgasta, mas se solta em lascas. Nesta forma, o esmalte apresenta manchas opacas com coloração que varia do branco ao castanho, passando pelo amarelo e pelo avermelhado, e radiograficamente apresenta densidade semelhante ou inferior à dentina. ${ }^{1,4}$

Em decorrência dos defeitos na formação do esmalte, os pacientes afetados podem apresentar sensibilidade dentária, perda da dimensão vertical e comprometimento estético.5,6

A prevalência da AI na população mundial é de um em cada 14 mil indivíduos, sendo a hipocalcificada a forma de maior incidência, seguida pelos tipos hipomaturada e hipoplásica. A definição do diagnóstico da AI não é fácil, em razão de a doença apresentar características clínica não especificas, podendo ser confundida com outras anomalias do esmalte dentário, como a hipoplasia de esmalte e a fluorose dental. Dessa forma, é muito importante que o cirurgião-dentista esteja preparado para reconhecer, diagnosticar e tratar precocemente essa alteração.7

A dificuldade no diagnóstico, a baixa qualidade estética, a alta sensibilidade e o comprometimento das propriedades mecânicas relacionadas a essa anomalia do esmalte causam a redução da motivação do paciente, representando um dos principais desafios para o cirurgião-dentista. Dessa forma, no tratamento da AI se faz necessária uma abordagem multidisciplinar, que tende a se estender por um longo período, e lide diretamente com aspectos psicológicos da qualidade de vida do paciente. ${ }^{8}$

Em razão de os diferentes tipos de AI apresentarem características clínicas semelhantes, é necessário muito cuidado para chegar ao diagnóstico correto, sendo de extrema importância o conhecimento das suas diferentes formas pelo profissional, associado a um correto exame clínico e radiográfico. ${ }^{9}$

Do ponto de vista odontológico, o tratamento dessas anomalias tem como principal objetivo aperfeiçoar a qualidade da saúde oral do paciente, ou seja, melhorar a estética, aliviar a dor ou o incômodo provocado pela dentina exposta e prevenir a atrição, levando sempre em consideração o grau de escurecimento dentário e o desconforto 
causado ao paciente. O tratamento da AI é dividido três fases. Inicialmente, a fase de prevenção visa instruir o paciente acerca da higiene oral e da aplicação tópica de flúor, remover o tecido cariado, caso exista, e realizar restaurações com cimento ionômero de vidro. Após essa etapa, inicia-se a fase restauradora, na qual serão realizadas restaurações em resinas compostas, resinas indiretas, facetas em cerâmica ou metalocerâmicas, considerando o caso, a expectativa e a idade do paciente. Por fim, a fase de preservação, de tempo indeterminado, tem o objetivo de garantir estabilidade estética e funcional ao paciente. ${ }^{10}$

Este trabalho visa relatar as características clínicas de um caso de AI do tipo IIA (hipomaturado com pigmentação difusa), apresentando as formas de tratamento utilizadas e seus impactos na qualidade de vida do paciente.

\section{RELATO DE CASO}

O responsável pelo paciente assinou a autorização de uso de imagens.

Um paciente com leucoderma, de 7 anos de idade, do sexo masculino e de classe média, residente na cidade de Araxá, município de Minas Gerais, foi encaminhado à Policlínica Getúlio Vargas, da Universidade de Uberaba, para realizar um tratamento odontológico. Ele se queixava de dentes escurecidos, sensibilidade dentária e ter procurado vários profissionais que não conseguiram solucionar o problema. Na anamnese não foi registrada nenhuma alteração sistêmica, e a mãe não relatou casos parecidos na família, nem intercorrências durante a gestação.

O exame físico extraoral demonstrou que o paciente apresentava desenvolvimento físico e mental normais. Era evidente que a pigmentação dos olhos, dos cabelos e da pele do paciente eram muito claras, não sendo compatível com a dos demais familiares. No exame físico intraoral observou-se que todos os dentes, tanto da dentição decídua quanto da permanente, apresentavam alteração de cor (amarelo acastanhado), superfície rugosa, presença de cálculos dentais nos incisivos inferiores e mordida topo a topo. O esmalte era clinicamente friável e se destacava da dentina em lascas, causando perda prematura do esmalte e sensibilidade dentinária exacerbada. Ademais, não foi possível detectar lesão de cárie nas dentições decíduas e permanentes (Figura 1).

No exame radiográfico (Figura 2) foi possível observar que o volume dos tecidos dentários estava normal, mas que havia falta de contraste entre esmalte e dentina em todos os dentes. O estágio de formação dos dentes permanentes ainda não erupcionados estava morfologicamente e cronologicamente normal, porém com ausência de cripta óssea de terceiros molares inferiores e superiores.

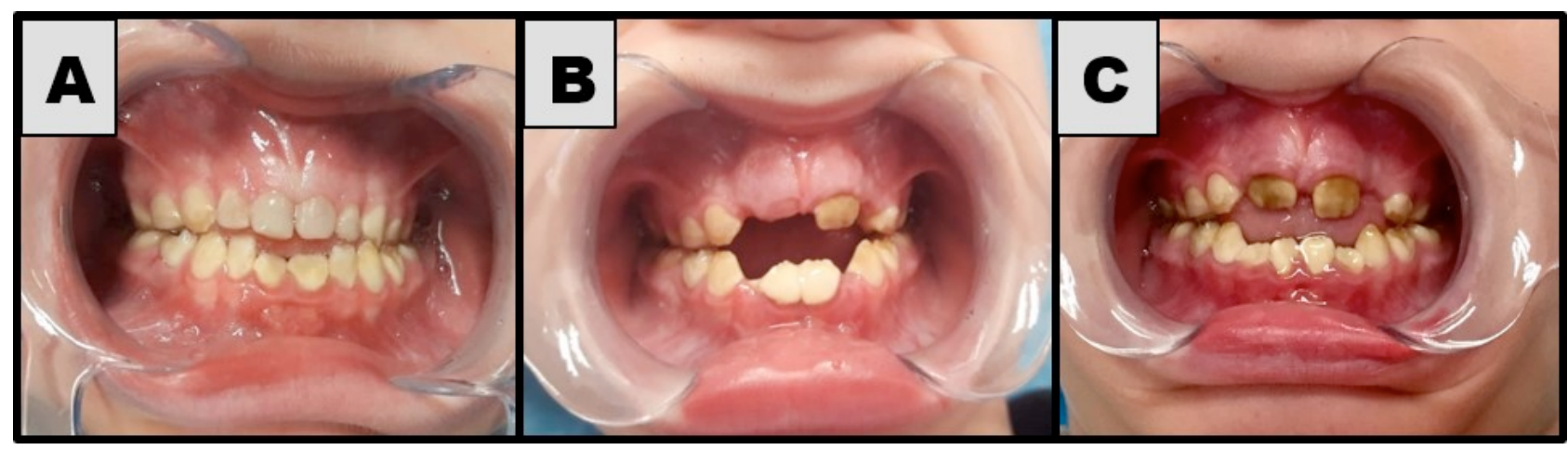

FIGURA 1 | A: dentição decídua; B: cálculos dentais nos incisivos inferiores e mordida topo a topo; C: incisivos permanentes em erupção. 


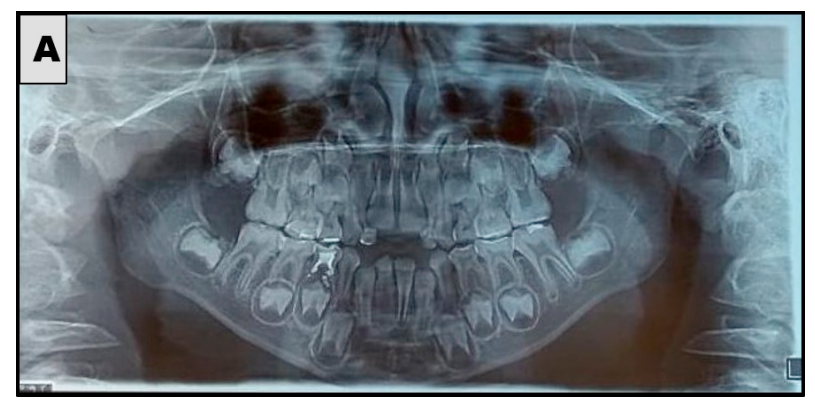

FIGURA 2 | Radiografia panorâmica.

Com base na anamnese e nos exames físicos, o paciente foi diagnosticado com amelogênese imperfeita do tipo IIA (Phenotype OMIM number: 615887). A mãe foi orientada e informada sobre a origem hereditária/genética, as características e as formas de tratamento dessa anomalia.

Como o paciente apresentava dentes ainda em erupção, optou-se por um tratamento conservador, feito por meio de restaurações com cimento de ionômero de vidro (Maxxion R, FGM, Joinville-SC, Brasil) (Figura 3), aplicação de flúor verniz (Fluorniz, SS White, Rio de Janeiro-RJ, Brasil) em cada sessão e bochechos com fluoreto de sódio 0,05\% diariamente (manipulado).

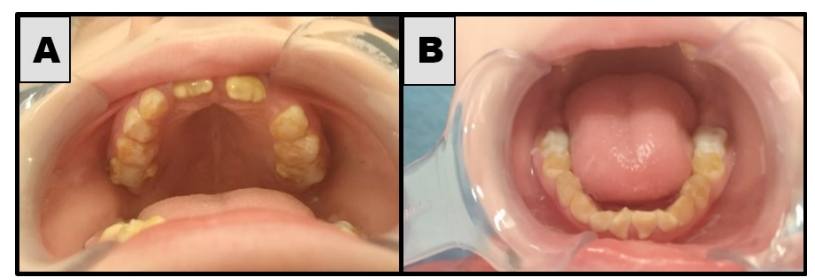

FIGURA 3 | A: aplicação de cimento de ionômero de vidro nos primeiros molares permanentes na maxila; B: aplicação de cimento de ionômero de vidro nos primeiros molares permanentes na mandíbula.

Durante o acompanhamento clínico, foi instalado aparelho ortopédico funcional pista indireta planas simples (PIPS) a fim de estimular o crescimento transversal, fazer o alinhamento dos elementos e conseguir espaço para os incisivos laterais superiores permanentes (Figura 4).

Durante as primeiras consultas, foi possível notar que o paciente se queixava de problemas socioafetivos e estéticos por causa das alterações dentárias e sistêmicas. Para medir o impacto dessas alterações na qualidade de vida do paciente foi aplicado o Questionnaire for measuring child oral health-related quality of life, conforme proposto por Jokovic et al. ${ }^{11}$, antes e depois do tratamento. A Tabela 1 apresenta os resultados encontrados.

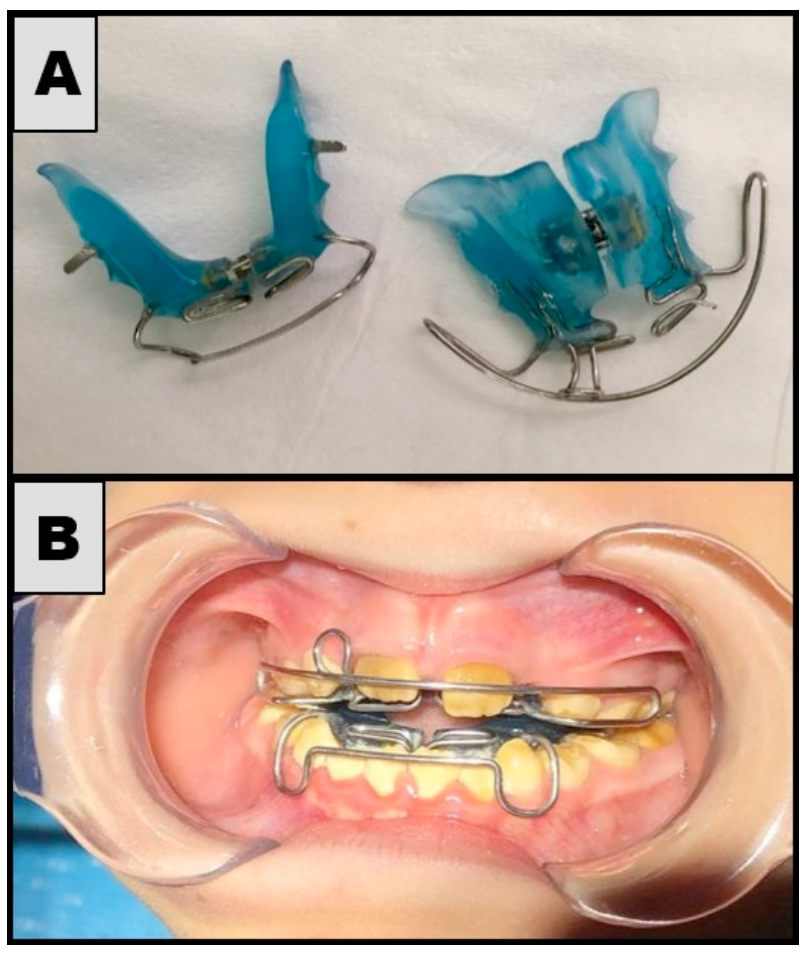

FIGURA 4 | A: aparelho ortopédico funcional do tipo pista indireta planas simples; B: paciente utilizando aparelho ortopédico funcional.

TABELA 1 | Pontuação geral e pontuações das subescalas do Questionnaire for measuring child oral health-related quality of life, proposto por Jokovic et al. ${ }^{11}$

\begin{tabular}{c|c|c}
\hline Dados avaliados & $\begin{array}{c}\text { Antes do } \\
\text { tratamento }\end{array}$ & $\begin{array}{c}\text { Depois do } \\
\text { tratamento }\end{array}$ \\
\hline Sintomas orais & 8 & 0 \\
\hline $\begin{array}{c}\text { Limitações } \\
\text { funcionais }\end{array}$ & 15 & 2 \\
\hline $\begin{array}{c}\text { Bem-estar } \\
\text { emocional }\end{array}$ & 20 & 2 \\
\hline Bem-estar social & 13 & 4 \\
\hline Escala total & 56 & 8 \\
\hline
\end{tabular}

Com o avanço do tratamento, foi possível notar grande melhora na dor do paciente, o que contribuiu de forma direta para sua autoestima, 
sua alimentação, sua higiene e seu comportamento, considerando suas características clínicas.

\section{DISCUSSÃO}

As características sociodemográficas da amelogênese imperfeita variam de acordo com a localização geográfica, com prevalência de 1:700 na Suécia e 1:14.00o nos Estados Unidos, por exemplo. ${ }^{12}$ A AI autossômica recessiva, a AI hipoplásica autossômica dominante e a AI autossômica recessiva hipomaturada são as mais comuns, não apresentando diferença entre sexos. ${ }^{13,14}$

O paciente do estudo apresenta AI do tipo hipomaturado com padrão de herança recessivo, dado encontrado em $43 \%$ dos indivíduos acometidos por AI no estudo de Farias et al. ${ }^{13}$ Ademais, o paciente foi diagnosticado com maloclusão, condição que acometia 41 dos 75 (54,7\%) pacientes descritos no estudo de Ceyhan, Kirzioglu e Emek. ${ }^{14}$

Mediante a anamnese e os exames físico e radiográfico, confirmou-se o diagnóstico de AI do tipo IIA no caso apresentado. A coloração fosca exibida pelo esmalte na dentição decídua e amarelo-acastanhado nos dentes permanentes representa deformidade ocorrida durante sua formação, clinicamente observado na estrutura do elemento dental. Essas características auxiliaram no direcionamento do diagnóstico, uma vez que elas já foram relatadas em estudos anteriores. ${ }^{1,3,4}$

O tratamento da AI é muito diverso, pois depende do tipo e da gravidade da desordem para se realizar um plano de tratamento adequado. Nos pacientes jovens, a finalidade do tratamento é manter a maior quantidade de estrutura dentária possível até que eles atinjam uma idade em que possam optar por técnicas menos conservadoras para a reabilitação dental..$^{15,16}$

Um tratamento restaurador conservador da amelogênese imperfeita é indicado para diversos casos, como de crianças e jovens, e pacientes de baixo nível socioeconômico, devido ao menor custo do tratamento, ao comprometimento estético e funcional suave, à alteração de cor superficial no esmalte, à sensibilidade dentária e como tratamento intermediário. É contraindicado em casos de comprometimento pulpar, grande alteração de cor e forma dos dentes, comprometimento estético e funcional severo, desgaste excessivo do esmalte, problemas oclusais (mordida aberta anterior e posterior, perda de dimensão vertical) e perdas múltiplas de elementos dentários. ${ }^{17}$

De acordo com Cocco et al., ${ }^{18}$ a escolha de tratamento para pacientes jovens seria a técnica adesiva direta, pois conserva a estrutura dental, é capaz de restaurar a função e proporciona uma aparência estética melhor - visto que, em geral, os pacientes com AI sofrem uma experiência estética negativa -, propiciando, assim, um efeito psicossocial positivo. Em contrapartida, Bogosavljevic et al. ${ }^{19}$ afirmam que a restauração protética fixa com coroas envolve perda severa da estrutura dentária e deve ser utilizada como tratamento final nos pacientes com AI, momento no qual já se realizaram tentativas com métodos conservadores.

Para os casos em que os pacientes apresentam dentição decídua e/ou mista, a abordagem clínica deve ser minimamente invasiva, o ionômero de vidro deve ser a opção nesses casos, pois trata-se de um material com biocompatibilidade à estrutura dental, liberação de flúor e de técnica fácil. A aplicação em um único incremento agiliza o procedimento, o que deve ser levado em consideração ao tratar pacientes pediátricos. ${ }^{20}$

Apesar das múltiplas opções terapêuticas para reabilitar pacientes portadores de AI, que vão desde sessões de aplicação de verniz fluoretado até a confecção de coroas totais, o tratamento definitivo deve ser feito após o restabelecimento da dimensão vertical de oclusão, estabilidade oclusal, higiene oral adequada, completa erupção dentária permanente e término do crescimento. ${ }^{21}$

Os padrões de beleza atualmente estão cada vez mais relacionados à estética dentária; sorrisos 
harmônicos cada vez mais "brancos" são solicitados por pacientes de diversas faixas etárias, especialmente pelos mais jovens, o que influencia diretamente a autoestima e o convívio social de pacientes com AI. ${ }^{22}$

De acordo com Figueiredo et al., ${ }^{16}$ o impacto negativo provocado pela AI na qualidade de vida da criança e do adolescente é muito relevante, não apenas pelo comprometimento estético, mas também pelo desconforto causado pela sensibilidade dentária, fonética alterada, alteração facial, dieta alimentar errada ou insuficiente, problemas periodontais precoces e, na maioria dos casos, atraso na erupção. Considerando os efeitos psicossociais desses impactos desfavoráveis, o diagnóstico precoce e o acompanhamento multidisciplinar são de extrema importância para que se consiga obter sucesso no tratamento dos portadores de amelogênese imperfeita.

Durante este estudo foi possível observar a mudança na qualidade de vida do paciente após o tratamento. As sessões com o uso do cimento de ionômero de vidro, juntamente com as aplicações de flúor, reduziram $100 \%$ da sensibilidade dentinária da qual o paciente se queixava.

\section{CONCLUSÃO}

O conhecimento dos diferentes tipos de AI pelo profissional é de extrema importância para o correto diagnóstico. O tratamento da AI proposto no caso clínico apresentado foi capaz de devolver a saúde bucal do paciente e, consequentemente, reintegrá-lo ao convívio social com maior qualidade de vida.

\section{REFERÊNCIAS}

1. Azevedo MS, Goettems ML, Torriani DD, Romano AR, Demarco FF. Amelogenesis imperfecta: clinical aspects and treatment. Rev Gauch Odontol. 2013;61(1 Suppl):491-6.

2. Witkop CJ Jr. Amelogenesis imperfecta, dentinogenesis imperfecta and dentin dysplasia revisited: problems in classification. J Oral Pathol. 1988;17(9-10):547-53. Doi: https://doi.org/10.1111/j.16oo-0714.1988.tbo1332.x.
3. Crawford PJM, Aldred M, Bloch-Zupan A. Amelogenesis imperfecta. Orphanet J Rare Dis. 2007;2:17. Doi: https:// doi.org/10.1186/1750-1172-2-17.

4. Beraldo CBS, Silva BJA, Valerio CS, Mazzieiro ET, Manzi FR, Cardoso CAA. Amelogenesis imperfecta: clinical case report. RFO UPF. 2015;20(1):101-4.

5. Morgado CL, Azul AC. A amelogénese imperfeita - uma revisão da literatura. Revista Portuguesa de Estomatologia, Medicina Dentária eCirurgia Maxilofacial. 2009;50(4):243-50. Doi: https://doi.org/10.1016/s1646-289o(o9)70025-1.

6. Couto ACF, Pupin MA, Dias MA, Dande JV, Barbosa D, Aleva LFG, et al. Amelogênese imperfeita: revisão da literatura. Revista Eletrônica Acervo Saúde. 2012;1 Supl:34-40.

7. Santos CT, Picini C, Czlusniak GD, Alves FBT. Anomalias do esmalte dentário - revisão de literatura. Arch Health Invest. 2014;3(4):74-81.

8. Trentesaux T, Rousset MM, Dehaynin E, Laumaillé M, Delfosse C. 15-year follow-up of a case of amelogenesis imperfecta: importance of psychological aspect and impact on quality of life. Eur Arch Paediatr Dent. 2013;14(1):47-51. Doi: https://doi.org/10.1007/s40368-012-0008-1.

9. Castagnoli TFB, Koubik ACGA. A importância dos aspectos clínicos e radiográficos da amelogênese imperfeita no auxílio do diagnóstico [trabalho de conclusão de curso]. Curitiba: Universidade Tuiuti do Paraná; 2013.

10. Borde BT, Araujo IRS, Valente AGLR, Tannure PN. Desafios no diagnóstico e tratamento da amelogênese imperfeita: relato de caso. Rev Odontol UNICID. 2018;30(2):216-22. Doi: https://doi.org/10.26843/ro_unicidv3022018p216-222.

11. Jokovic A, Locker D, Tompson B, Guyatt G. Questionnaire for measuring oral health-related quality of life in eight- to ten-year-old children. Pediatr Dent. 2004;26(6):512-8.

12. Neville BW, Damm DD, Allen CM, Bouquot JE. Patologia oral e maxilofacial. 3a ed. Rio de Janeiro: Elsevier; 2009.

13. Adorno-Farias D, Ortega-Pinto A, Gajardo P, Salazar A, Morales-Bozo I, Werlinger F, et al. Diversity of clinical, radiographic and genealogical findings in 41 families with amelogenesis imperfecta. J Appl Oral Sci. 2019;27:e20180359. Doi: https://doi.org/10.1590/1678-7757-2018-0359.

14. Ceyhan D, Kirzioglu Z, Emek T. A long-term clinical study on individuals with amelogenesis imperfecta. Niger J Clin Pract. 2019;22(8):1157-62. Doi: https://doi.org/10.4103/ njcp.njcp_227_18.

15. Coffield KD, Phillips C, Brady M, Roberts MW, Strauss RP, Wright JT. The psychosocial impact of developmental dental 
defects in people with hereditary amelogenesis imperfecta. J Am Dent Assoc. 2005;136(5):620-30. Doi: https://doi.org/ 10.14219/jada.archive.2005.0233.

16. Figueiredo RG, Moreira RF, Simões PS, Novaes SEA, Fonseca ACL, Miranda MS. Amelogenesis imperfecta: the importance of early diagnosis for adolescent health and development: Case report. Rev Adolesc Saude. 2016;13(2):87-93.

17. Silva FS, Lima VS. Tratamento restaurador conservador na amelogênese imperfeita: quando indicar essa opção? Rev Flum Odontol. 2018;(48):1-14. Doi: https://doi.org/ 10.22409/ijosd.v2i48.375.

18. Cocco AR, Baldissera RA, Lund RG, Martos J. Rehabilitation of dental function and aesthetics in a young patient presenting amelogenesis imperfect. J Clin Dent Res. 2017;14(2):72-82. Doi: https://doi.org/10.14436/2447-911x.14.2.072-082.oar.

19. Bogosavljević A, Misina V, Jordacević J, Abazović M, Dukić S, Ristić L, et al. Treatment of teeth in the esthetic zone in a patient with amelogenesis imperfecta using composite veneers and the clear matrix technique: A case report. Vojnosanit Pregl. 2016;73(3):288-92. Doi: https://doi.org/ 10.2298/vsp141125053b.

20. Ferreira SH, Pasini CM, Rodrigues PH, Cardoso MZ, Borges TS. Amelogenesis imperfecta in a child with cerebral palsy. Stomatos. 2017;23(44):13-23.

21. Ağaçkiran E, Tümen EC, Celenk S, Bolgül B, Atakul F. Restoring aesthetics and function in a young boy with hypomature amelogenesis imperfecta: A case report. ISRN Dent. 2011;2011:586854. Doi: https://doi.org/ 10.5402/2011/586854.

22. Holanda RC, Feitosa DMA, Gonçalves GKM, Alves KSS, Gouveia THN, Lobo PLD. Reabilitação estética e funcional em paciente com amelogênese imperfeita: relato de caso. Rev Fac Odontol Lins. 2017;27(2):45-52. Doi: https://doi.org/ 10.1560o/2238-1236/fol.v27n2p45-52. 